\title{
Marrow hypoplasia with splenomegaly in adolescence
}

\author{
JEFFREY H ROBERTSON, PHILIP BURNSIDE, AND DEIRDRE C SALMON \\ From the Department of Haematology, Belfast City Hospital, Belfast, Northern Ireland
}

SUMMARY Four adolescents presented with leucopenia, thrombocytopenia, and splenomegaly; three had associated congenital abnormalities and two had a cirrhosis. They provide further evidence of an association between hepatic disease and bone marrow dysfunction. It is considered that these patients suffer from a genetic or constitutional disorder which may result in a susceptibility of their bone marrow to hypoplasia following viral hepatitis or other infections.

In recent years there has been renewed interest in the pathogenesis of marrow hypoplasia. This has arisen partly from the observations of an association between marrow aplasia and infection with the hepatitis virus or other forms of liver disease. We describe here a disorder in four adolescents in which there was an association between bone marrow hypoplasia, which was probably genetically determined, and hepatic disease. The four patients were seen during the past 10 years, and all had leucopenia, thrombocytopenia, splenomegaly, and bone marrows which were hypoplastic and deficient, especially in megakaryocytes and granulopoietic elements. Two patients had cirrhosis, two were mentally retarded, and three had congenital abnormalities.

\section{Case reports}

CASE 1

This 15 -year-old girl was referred to this laboratory

Received for publication 21 January 1980 because a routine blood count had revealed a leucopenia and thrombocytopenia. She was symptomless; the only relevant medical history was an episode of presumed infective hepatitis after contact with a jaundiced neighbour when she was 13 years old. On examination she was a normally intelligent schoolgirl. There was no skin pigmentation, and the only abnormal finding was splenomegaly of $2 \mathrm{~cm}$ below the costal margin. The results of the main investigations are summarised in the Table. A blood count showed a mild normocytic anaemia, with leucopenia and thrombocytopenia. The ESR was $12 \mathrm{~mm} / \mathrm{h}$, the reticulocyte count was $2 \%$, and the direct Coombs test was negative. In the blood film there was a neutropenia; the red cells appeared normal. A bone marrow aspirate was rather poorly cellular, granulopoietic precursors were scanty, and erythropoiesis was macronormoblastic. Trephine biopsies were taken from three sites on the posterior iliac crests. These showed areas of fatty aplastic marrow separating cellular foci made up almost entirely of normoblasts (Fig. 1).

Main clinical and laboratory findings

\begin{tabular}{|c|c|c|c|c|}
\hline & \multicolumn{4}{|l|}{ Case } \\
\hline & $I$ & 2 & 3 & 4 \\
\hline $\begin{array}{l}\text { Age at presentation (yr) } \\
\text { Hb (g/dl) } \\
\text { MCV (fl) } \\
\text { WBC }\left(10^{\circ} / 1\right) \\
\text { Neutrophils }\left(10^{\circ} / 1\right) \\
\text { Platelets }\left(10^{\circ} / 1\right) \\
\text { Haptoglobins }(0 \cdot 3-5 \cdot 0 \mathrm{~g} / 1) \\
\text { Liver function tests AST (2-35) } \\
\text { Splenomegaly (cm) } \\
\text { Liver biopsy } \\
\text { Mental retardation } \\
\text { Familial case } \\
\text { Congenital abnormalities }\end{array}$ & $\begin{array}{l}15 \\
11 \cdot 0 \\
86 \\
2 \cdot 1 \\
0 \cdot 84 \\
67 \\
0 \cdot 20 \\
68 \\
2 \\
\text { Cirrhosis } \\
- \\
\text { Yes } \\
\end{array}$ & $\begin{array}{l}13 \\
14 \cdot 3 \\
100 \\
2 \cdot 4 \\
1 \cdot 58 \\
105 \\
0 \cdot 35 \\
\text { Normal } \\
1 \\
\text { Normal } \\
\text { Present } \\
\text { Yes } \\
\text { Yes }\end{array}$ & $\begin{array}{l}16 \\
12 \cdot 0 \\
98 \\
2 \cdot 7 \\
0 \cdot 97 \\
70 \\
0 \cdot 12 \\
49 \\
3 \\
\text { Normal } \\
- \\
\text { Yes }\end{array}$ & $\begin{array}{l}16 \\
12 \cdot 3 \\
91 \\
1 \cdot 5 \\
1 \cdot 00 \\
60 \\
0 \cdot 12 \\
\text { Normat } \\
2 \\
\text { Cirrhosis } \\
\text { Present } \\
\text { Yes }\end{array}$ \\
\hline
\end{tabular}




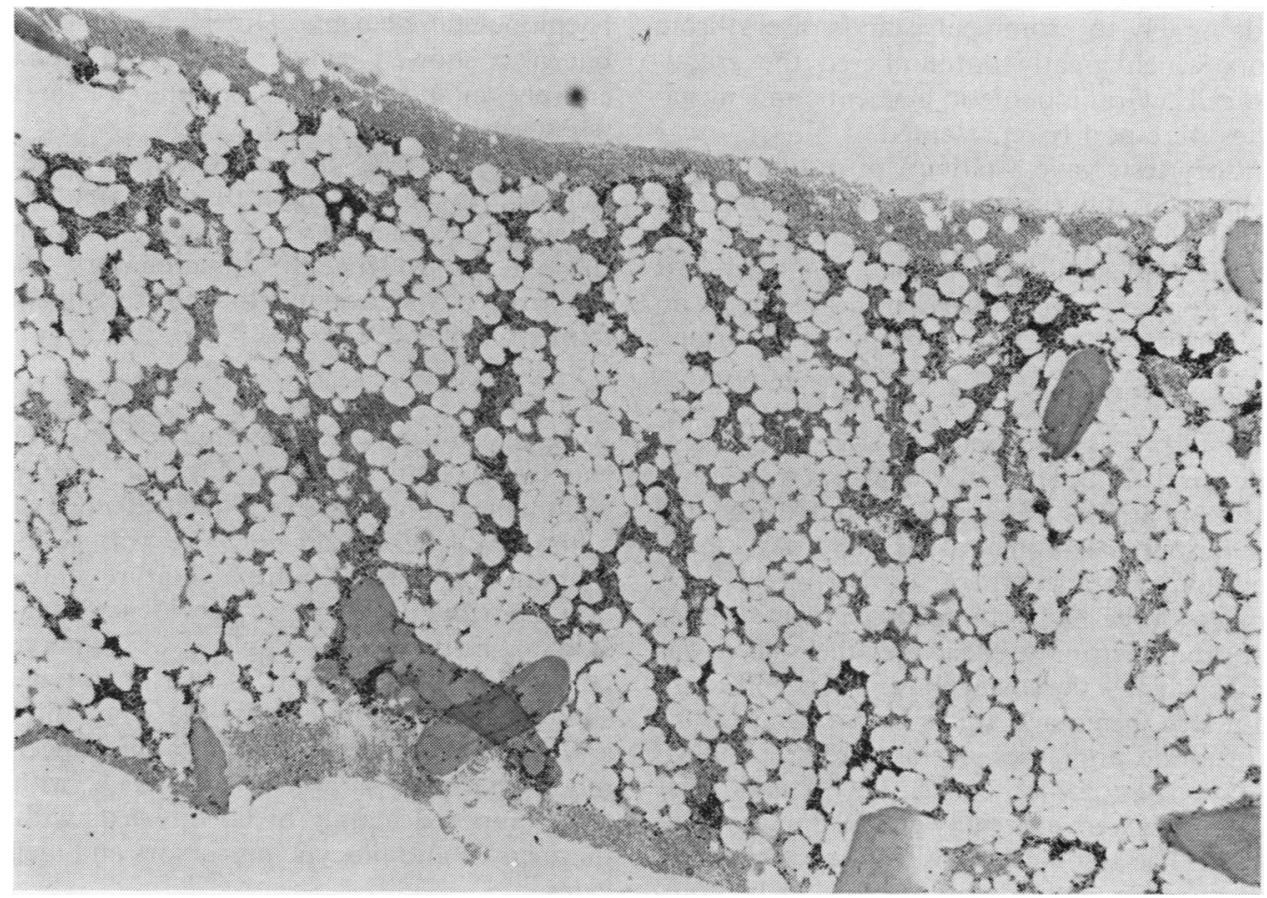

Fig. 1 Case 1. Bone trephine biopsy showing marked hypoplasia (Haematoxylin and eosin $\times 40$ ).

As the plasma haptoglobin was found to be low, a red cell survival using the patient's chromium labelled cells was performed but found to be normal; there was also no evidence of pooling of the labelled cells in the spleen, and measurement of plasma volume using ${ }^{131}$ I albumin was also normal. The acid lysis (Ham's) test was normal as was haemoglobin electrophoresis, there being no increase in $\mathrm{HbF}$. Chromosome studies on marrow samples revealed a normal female karyotype with no evidence of chromosome breaks. Liver function tests were normal apart from an elevated aspartate transaminase, and estimation of serum proteins revealed normal albumin and globulin levels although on electrophoresis there was a polyclonal increase in the gamma globulin. A needle liver biopsy demonstrated a macronodular cirrhosis without evidence of an inflammatory infiltrate or of recent regenerative changes in the liver cells. The serum was negative for Australia antigen. A 24-hour estimation of copper excretion gave a normal result, and the serum level of $\alpha_{1}$ antitrypsin was normal. As markers of an autoimmune disorder, antinuclear factor, smooth muscle and antimitochondrial antibodies and LE cells were looked for, but these tests were negative. A urinary amino acid chromatogram was also normal.
There was no history of congenital abnormality or liver disease in the family but a first cousin on her father's side had been investigated for a blood disorder without a diagnosis being reached. This patient is the subject of case 2 .

\section{CASE 2}

This 24-year-old man was a cousin of case 1 . Eleven years ago, aged 13 , he was investigated at another hospital because of excessive oozing after teeth extraction. It was noted that he was thrombocytopenic but a cause was not established. Since then he had been well, the only symptom being a tendency to bruise easily. He had been educationally subnormal, attending a special school. On examination several bruises were present on the legs, several congenital abnormalities were noted, the left testis was undescended and the right was in the inguinal canal, he had an anti-mongolian slant, high arched palate, malocclusion of the jaw, and pectus excavatum. The spleen was just palpable. A blood count revealed a mild thrombocytopenia and moderate neutropenia. There were no immature cells in the film. A bone marrow aspirate yielded a moderately cellular smear with macronormoblastic erythropoiesis. A trephine biopsy showed a hypocellular marrow. The cellularity was greater than in the patient's cousin (case 1), 
being due largely to prominent islands of erythroid precursors which greatly outnumbered the granulopoietic cells. Granulopoietic elements and megakaryocytes were poorly represented.

Laboratory tests gave a pattern of results similar to those in case 1 , differing only in that liver function tests were normal as was an electrophoresis of serum proteins. The plasma haptoglobin was also just within the normal range. A barium meal showed no evidence of varices, and a liver biopsy was normal.

CASE 3

A 16-year-old boy was referred by his general practitioner in 1977 because of epistaxis and purpura. There was no other history apart from occasional previous episodes of bronchitis. He had never been jaundiced and was a youth of normal intelligence. On examination he had prominent pigmentation of the chest, abdomen, and back, and the spleen was enlarged $2 \mathrm{~cm}$ below the costal margin. A blood count revealed a leucopenia and thrombocytopenia. Bone marrow aspirate and trephine biopsies were taken from both posterior iliac crests. The smears were hypocellular and were especially deficient in granulopoietic cells. The trephine biopsies were variable in appearance; some showed the marrow to be composed entirely of fat cells with no recognisable haemopoietic elements. Others were partly aplastic but also showed cellular areas composed almost entirely of normoblasts, granulopoietic elements being very scanty (Fig. 2).

Laboratory tests gave a pattern of results similar to those in case 1 , indicating a slightly elevated aspartate transaminase, although a liver biopsy showed no evidence of inflammatory change or cirrhosis. It was not possible to investigate further family members.

\section{CASE 4}

A 16-year-old boy was referred for investigation of leucopenia and thrombocytopenia. He was educationally subnormal and had attended a special school. When seen in 1969 he was well and on examination was of normal stature and physical development. There was no evidence of abnormal skin pigmentation. The spleen was enlarged $2 \mathrm{~cm}$ below the costal margin. A peripheral blood film showed an occasional normoblast but the red cells and leucocytes were normal in appearance. A bone marrow trephine showed aplastic areas, with cellular foci composed mainly of normoblasts with smaller numbers of granulocytic precursors and megakaryocytes.

Laboratory tests (see Table) revealed a pattern of

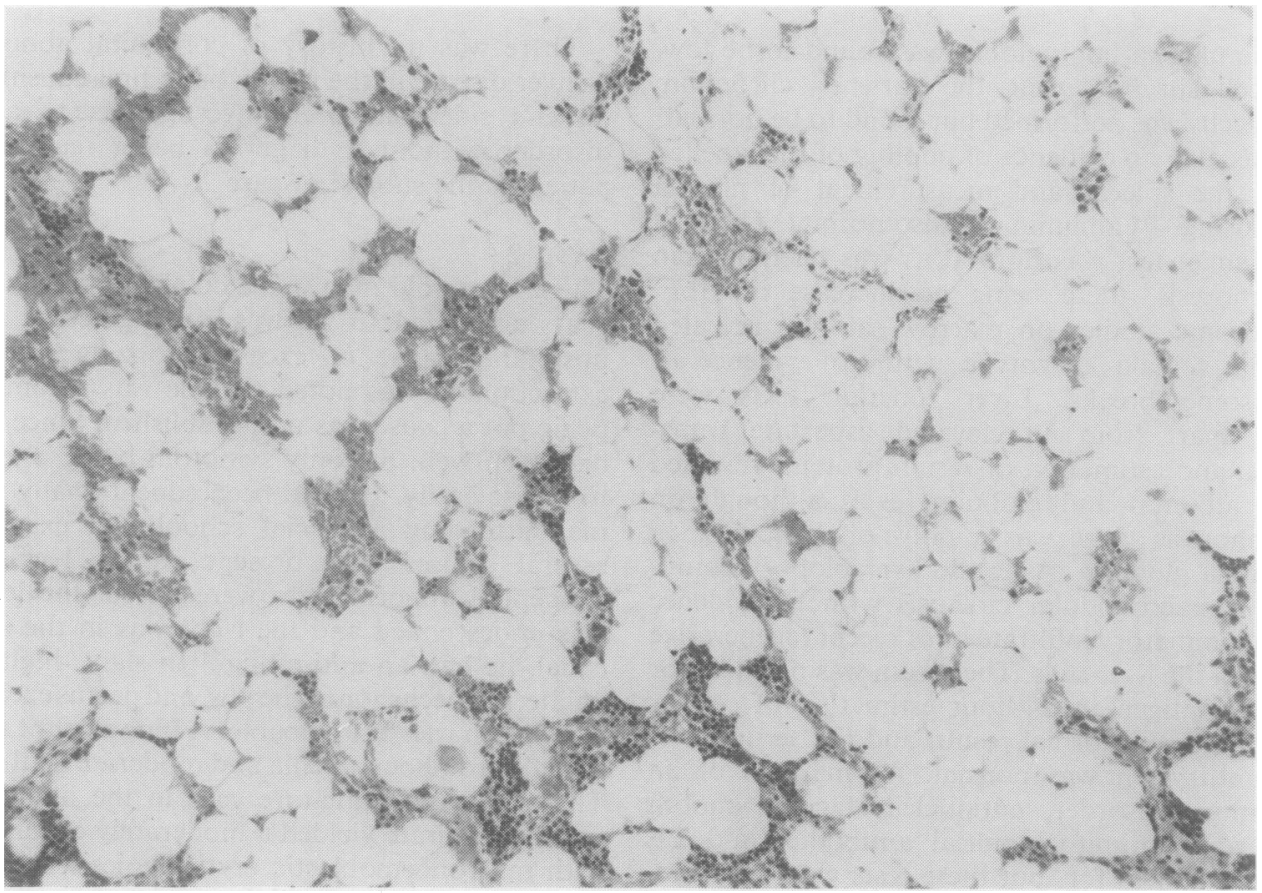

Fig. 2 Case 3. Bone trephine biopsy showing marked hypoplasia and islands of persisting normoblasts (Haematoxylin and eosin $\times 150)$. 
results similar to those in case 1 except that liver function tests were normal.

Two years after he was first seen he had a small haematemesis. Oesophageal varices were shown by a barium swallow, and liver biopsy demonstrated a macronodular cirrhosis with no evidence of active hepatitis. The liver function tests remained normal, as was a bromsulphalein excretion test. Further haematemesis required a portacaval shunt. It was possible to examine only the patient's mother who had a normal blood count.

\section{Discussion}

These four patients have a number of features in common. They all presented in adolescence and have splenomegaly and a hypoplastic marrow with leucopenia and thrombocytopenia but little or no anaemia. Three of the patients have decreased haptoglobins, and in the fourth the level was at the lower limit of normal. Mental retardation and cirrhosis were present together in one patient, and of the two familial cases, one had cirrhosis alone and the other had mental retardation, among other congenital abnormalities, suggesting that these two features are pathogenetically related (see Table).

Initially, thrombocytopenia, leucopenia, and splenomegaly were thought to be in keeping with hypersplenism. However, isotope studies showed no evidence of splenic pooling, increased plasma volume, or decreased red cell survival, and although selective splenic sequestration can occur, its presence in all four patients is unlikely. Furthermore, in two patients no condition that might lead to hypersplenism could be identified. The presence of marrow hypoplasia is also strongly against a hypersplenic mechanism, this disorder being invariably associated with a normally cellular or hyperplastic marrow. ${ }^{1}$

Our findings are more suggestive of an hereditary or constitutional marrow hypoplasia. The dominant feature of each marrow biopsy was areas of aplasia with scattered hyperplastic foci composed mainly of erythroid cells. Such hyperplastic areas are frequently seen in aplastic anaemia. ${ }^{2}$ Dyserythropoiesis is also a feature of aplastic anaemia and has been especially associated with constitutional cases. Indeed, it has been claimed that a low serum haptoglobin is a feature distinguishing constitutional from acquired aplastic anaemia. ${ }^{3}$ Other features indicating the congenital and probably hereditary nature of this disorder are the two cousin cases and the finding that three of the patients were also affected by congenital abnormalities including mental retardation and excessive skin pigmentation. Such abnormalities are a feature of other constitutional disorders of marrow function such as Fanconi's anaemia, congenital hypoplastic anaemia, and amegakaryocytic thrombocytopenia. ${ }^{4-6}$

It is, however, difficult to identify our patients with one of the previously described syndromes. They most resemble Fanconi's anaemia, although cases of this disorder rarely present after the age of 10 years. Again, this disorder generally results in pancytopenia, and although it may occasionally produce a hypoplasia of only one or two cell lines, such a presentation in all our four patients would be very unusual. Most cases of Fanconi's anaemia also show an increase in fetal haemoglobin and characteristically chromosomal breaks in bone marrow preparations, ${ }^{46}$ neither being present in any of our patients.

The presence of cirrhosis in two patients is of interest in view of the well established but still quite obscure relationship between disease of the bone marrow and liver. Aplastic anaemia has followed hepatic damage by a number of drugs, some of which are not normally myelotoxic, and, more recently, the occasional development of aplastic anaemia following viral hepatitis has been recognised. In a review of the subject, it was considered that functional hepatic defects, whatever their cause, may lead to haemopoietic abnormalities. ${ }^{7}$ It was suggested that subclinical hepatitis might be responsible for a significant number of cases of idiopathic aplastic anaemia. The mechanism relating hepatitis and marrow aplasia is unknown but a number have been proposed, including initiation of an autoimmune reaction against haemopoietic cells, failure of a damaged liver to provide nutrients for the marrow or to detoxify myelotoxic metabolites, or possibly an individual susceptibility on an unexplained basis.?

In our patients, it is possible that the cirrhosis might in some way be a further facet of the other congenital abnormalities but we are unaware of liver disease having been previously described in association with other constitutional bone marrow disorders. We were able to exclude an associated $\alpha_{1}$ antitrypsin deficiency or an abnormality in copper metabolism in any of the patients. On the other hand, the history of an illness strongly resembling viral hepatitis in case 1 suggests that the liver disease was acquired. Although there is no indication whether this patient's peripheral blood was normal before the episode of hepatitis there is evidence that genetic factors can dictate a haemopoietic suppression following a wide range of infections. ${ }^{8}$ Two siblings have also been described who both developed red cell aplasia during the course of hepatitis. ${ }^{9}$ It is possible, therefore, that our patients have a constitutional disorder reflected by varying congenital abnormalities and also a liability to develop persistent marrow hypoplasia with leucopenia and 
thrombocytopenia following hepatitis or perhaps other infections.

These patients are of interest because they may direct attention to other young patients with hepatic disease, splenomegaly, and a peripheral blood cytopenia due, not to an expected hypersplenism but to marrow hypoplasia. Further studies on such patients may shed light on the mechanism that brings about the fulminant aplasia which sometimes follows viral hepatitis.

\section{References}

1 Hardisty RM, Weatherall DJ, eds. Blood and its Disorders. Oxford: Blackwell, 1974;1228.

2 Kansu E, Erslev A. Aplastic anaemia with 'hot pockets'. Scand J Haemat 1976;17:326-39.

${ }^{3}$ O'Gorman-Hughes DW. The varied pattern of aplastic anaemia in childhood. Aust Pediatr $J$ 1966;2:228-36.
${ }^{4}$ Bloom GE. Disorders of bone marrow production. Pediatr Clin N Amer 1972;19:983-1008.

${ }^{5}$ Diamond LK, Allen DM, Magill FB. Congenital (erythroid) hypoplastic anaemia. A 25-year study. Amer $J$ Dis Child 1961;102:403-15.

- O'Gorman-Hughes DW. Aplastic anaemia in childhood. III. Constitutional aplastic anaemia and related cytopenias Med J Aust 1974;1:519-26.

7 Hagler L, Pastore RA, Bergin JJ. Aplastic anaemia following viral hepatitis. Medicine (Baltimore) 1975;54:139-64.

${ }^{8}$ Hilkowitz G. The aplastic crisis and erythroid maturation defect occurring simultaneously in three members of a family. Arch Intern Med 1960;105:100-8.

- Sears DA, George JN, Gold MS. Transient red cell aplasia in association with viral hepatitis: occurrence four years apart in siblings. Arch Intern Med 1975;135:1585-9.

Requests for reprints to: Dr JH Robertson, Department of Haematology, The Laboratories, Belfast City Hospital, Lisburn Road, Belfast 9, Northern Ireland. 